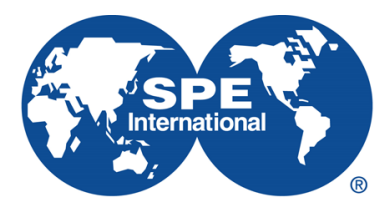

Society of Petroleum Engineers

\title{
SPE-200651-MS
}

\section{Implementation of Three-Phase Black-Oil Reservoir Models Assisted by Micro-Scale Analyses}

\author{
Ehsan Ranaee and Gaël Raymond Guédon, Department of Energy, Politecnico di Milano; Leili Moghadasi, \\ Eni SpA; Fabio Inzoli, Department of Energy, Politecnico di Milano; Monica Riva, Department of Civil and \\ Environmental Engineering, Politecnico di Milano; Giuseppe Maddinelli and Martin Bartosek, Eni SpA; Alberto \\ Guadagnini, Department of Civil and Environmental Engineering, Politecnico di Milano
}

Copyright 2020, Society of Petroleum Engineers

This paper was prepared for presentation at the SPE Europec featured at 82nd EAGE Conference and Exhibition originally scheduled to be held in Amsterdam, The Netherlands, 8 - 11 June 2020. Due to COVID-19 the physical event was postponed until 8 - 11 December 2020 . The official proceedings were published online on 8 June 2020.

This paper was selected for presentation by an SPE program committee following review of information contained in an abstract submitted by the author(s). Contents of the paper have not been reviewed by the Society of Petroleum Engineers and are subject to correction by the author(s). The material does not necessarily reflect any position of the Society of Petroleum Engineers, its officers, or members. Electronic reproduction, distribution, or storage of any part of this paper without the written consent of the Society of Petroleum Engineers is prohibited. Permission to reproduce in print is restricted to an abstract of not more than 300 words; illustrations may not be copied. The abstract must contain conspicuous acknowledgment of SPE copyright.

\section{Abstract}

Objective/Scopes: We aim at developing a viable workflow for the characterization of reservoir responses under Water Alternating Gas (WAG) conditions for enhanced oil recovery. We do so through a numerical Monte Carlo (MC) framework and by relying on $(i)$ a classical approach, which is grounded on employing results from laboratory-scale core-flooding experiments or ( $i i)$ an approach based on relative permeability curves inferred from pore-scale numerical simulations. In these settings we investigate $(i)$ the way uncertainties associated with the parameters of a reservoir model estimated through these approaches propagate to target modeling goals and (ii) assess (through Global Sensitivity Analyses) the relative importance of the uncertain quantities controlling the reservoir behavior via given model outcomes.

Methods/Procedures: We consider uncertainty in $(a)$ porosity and absolute permeability as well as $(b)$ parameters of relative permeability models. Three scenarios are assessed, accounting for spatial distribution of porosity and absolute permeability with differing degrees of complexity and corresponding to $(i)$ homogeneous; ( ii) randomly heterogeneous; and (iii) well-connected randomly heterogeneous fields. Spatial realizations of the heterogeneous fields are generated considering Gaussian random fields with a Gaussian kernel variance driving the degree of spatial correlation. The two modeling approaches considered take advantage of two-phase relative permeability curves, which are interpreted via commonly used models with uncertain parameters. Three-phase relative permeabilities are then characterized through a previously developed and tested sigmoid-based oil relative permeability model by taking into account hysteretic behavior of gas relative permeability. All field-scale simulations are performed on a simple reservoir model and are set within the MRST suite.

Results/Conclusions: In the case of a homogeneous reservoir, we note that reservoir simulation responses are strongly sensitive to the degree of convexity of the two-phase relative permeability curves. In the case of heterogeneous reservoir settings, results are almost similarly sensitive to porosity, characteristics of the 
relative permeability model, and the degree of heterogeneity of the reservoir. In the case of well-connected (randomly) heterogeneous fields, the importance of the porosity is stronger than in the heterogeneous setting lacking well connected regions.

Novel/Additive Information: Characterization of reservoir model attributes relying on pore-scale simulation approaches in the presence of uncertainty can provide a robust term of comparison which can be integrated within a classical reservoir simulation approach relying on relative permeability data stemming from core-flooding experiments. Our results document that uncertainties in the evaluation of $(i)$ reservoir model petrophysical attributes (porosity/permeability) and (ii) relative permeability model parameters can differently influence field-scale simulation outputs, depending on the degree of spatial heterogeneity of the reservoir.

Keywords: Reservoir simulation, pore-scale analyses, heterogeneity, relative permeability, uncertainty assessment

\section{Introduction}

Enhanced oil recovery (EOR) processes may often involve simultaneous flow of two or three immiscible fluids across a reservoir. A key aim of our study is the development of a viable workflow for the assessment of three-phase flow in porous systems with specific interest to water-alternating-gas (WAG) enhanced oil recovery processes.

Responses of a reservoir simulation model to the implementation of a WAG protocol depend on the characterization of (i) petrophysical rock properties (i.e., permeability, porosity), (ii) fluid-fluid and fluidsolid interactions in the system (as expressed through, e.g., relative permeability, capillary pressure), and (iii) heterogeneous distribution of reservoir hydrofacies (giving rise to preferential flow paths).

Previous works suggest that an increase in either porosity or permeability can lead to enhanced displacement efficiency (Christensen et al., 2001). Hence, documented failures of some WAG projects (even in the presence of reasonably high values of permeability/ porosity) have been attributed to the spatial heterogeneity of the reservoir properties (e.g., Afzali et al., 2018 and references therein). The efficiency of a WAG injection protocol can also be markedly influenced by the selected injection rate, the local flow rate in each (generally heterogeneous) region of the reservoir being proportional to the local ability of the system to convey flow (i.e., the local conductivity). Injectivity issues may then take place in the presence of high injection rates due to $(i)$ local contrasts across permeability and porosity values and/or (ii) the occurrence of high permeabilities along well-connected paths or fractures (Moghadasi et al., 2020). In this context, gas fingering and, consequently, early gas breakthrough might be associated with reservoir heterogeneities, such as fractures and occurrence of highly permeable zones (e.g., Afzali et al., 2018). In low permeability heterogeneous reservoirs, water flooding might be associated with issues related to poor injectability, low production rate, high water-oil ratio, and low oil recovery factor. Additionally, we note that a precise evaluation of relative permeabilities is critical to properly quantifying dynamics of multi-phase flow in oil- and gas- bearing formations.

Here, we focus on the appraisal of the joint role of petrophysical rock properties, relative permeability model, and reservoir heterogeneity on WAG practices. We do so by illustrating the results of combining information stemming from laboratory-scale investigations of multiphase flow on a Portland reservoir core sample with those collected through direct pore-scale simulations and employing the ensuing information to simulate WAG injection at reservoir scale.

Our evaluations of two-phase relative permeabilities rest here on two diverse approaches. Hereafter, we term as classical approach the procedure according to which field-scale simulations are grounded on the direct use of the results of (two-phase) coreflooding experiments. Otherwise, we term numerical approach the methodology based on the use of results from pore-scale simulations. 
The general layout of the analysis workflow is illustrated in Fig. 1 and includes the following steps:

1) assessment of two-phase water-oil (WO) and oil-gas (OG) relative permeability curves from $(a)$ laboratory experiments (classical approach) or (b) pore-scale simulations (numerical approach);

2) evaluation of the estimation variance of a set of controlling parameters and the width of the ensuing confidence interval for each of these;

3) setup a reservoir model for a collection of $\mathrm{N}$ Monte Carlo (MC) simulations of flow;

4) numerical simulation of the multi-phase flow field across the reservoir model;

5) post-processing of simulation outputs to obtain key statistics of the responses of the reservoir;

6) evaluation of the sensitivity of the simulation responses to the controlling parameters;

7) assessment of the uncertainties associated with simulation results.

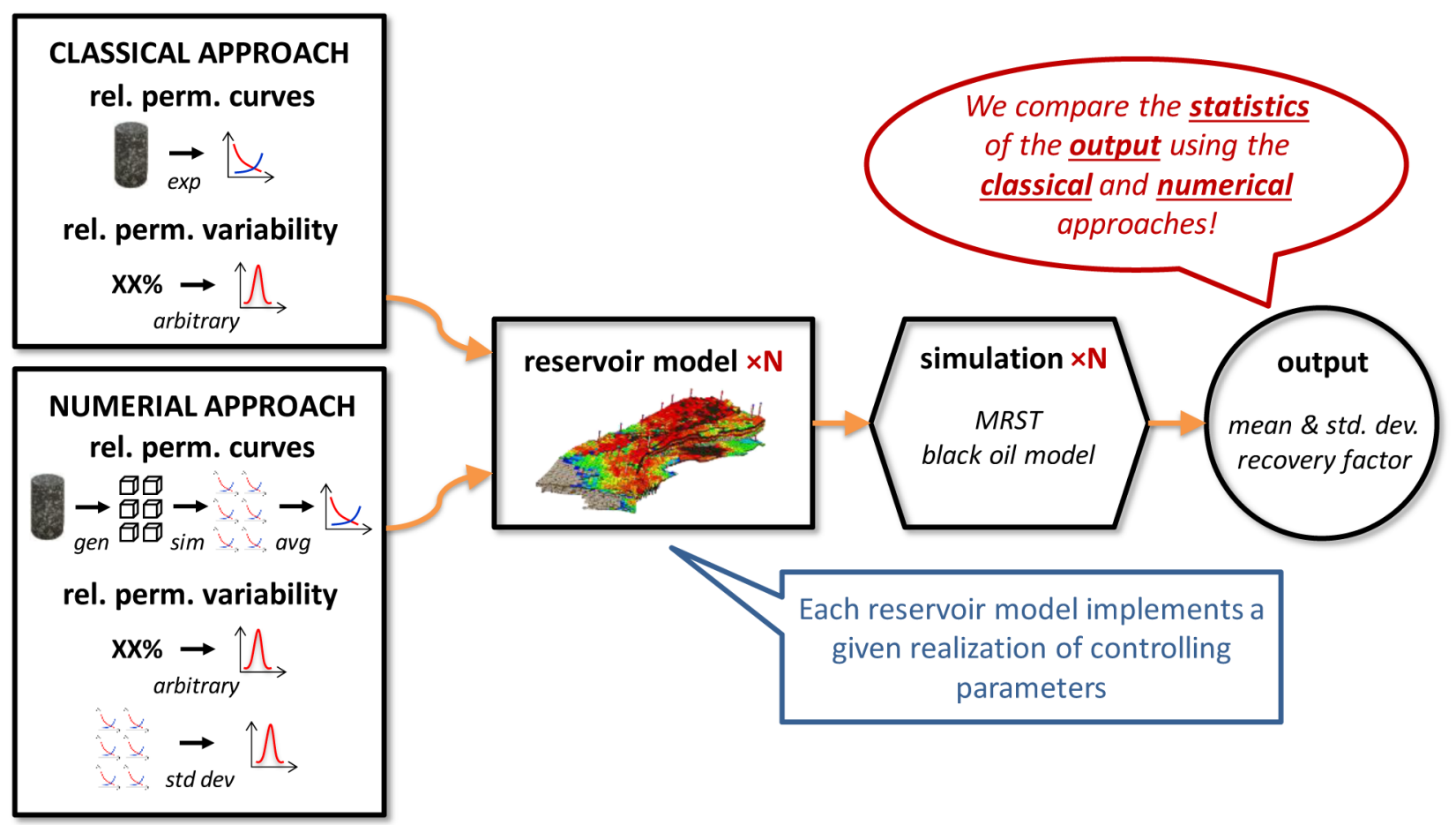

Figure. 1-Sketch of the workflow of the analysis ( $\mathrm{N}$ corresponds to the number of Monte Carlo simulations).

As uncertain model parameters, we consider: $(a)$ porosity and absolute permeability as well as $(b)$ parameters of the relative permeability (curves) model we employ. We then implement the classical approach upon considering that the selected input variables (controlling parameters) of the reservoir simulation model be associated with a constant $25 \%$ coefficient of variation, their estimates being assessed through a single core-flooding experiment on a Portland rock sample. Hereafter, we term these estimates as measurement-based information.

When considering the results of direct pore-scale two-phase flow simulations (i.e., our numerical approach), we evaluate uncertainties on reservoir model input parameters through a standard Maximum Likelihood (ML) framework grounded on results from computational fluid dynamics (CFD) simulations on three-dimensional (3-D) models (Guédon et al., 2019).

We set up a two-dimensional (horizontal) reservoir model modified from the original Odeh (1981) model as a test case for our reservoir-scale simulations under WAG. Uncertainties of the controlling parameters are then propagated to system responses through MC simulations.

Three scenarios have been considered, structured according to spatial distributions of porosity and absolute permeability fields with differing degrees of complexity. These correspond to a $(a)$ homogeneous, 
(b) heterogeneous, and (c) heterogeneous and well-connected system. The heterogeneous fields are based on the generation of Gaussian random fields with a Gaussian kernel variance that drives the correlation length of the field (see Hyman and Winter (2014) for details). Field with a marked degree of connectivity between high permeability values are obtained through the approach suggested by Zinn and Harvey (2003).

Sensitivity of the reservoir simulation responses to the variation of system input quantities are evaluated within a Global Sensitivity Analysis (GSA) framework (see e.g., amongst others, Saltelli et al., 2008; Dell'Oca et al., 2017; Bianchi Janetti et al., 2019 and references therein). Selected reservoir responses include time evolutions of the ( i) field average pressure, FPR, ( $i$ i) water-cut, FWCT, (iii) gas oil ratio, FGOR, (iv) oil recovery, FOR, and (v) net-present-value, NPV (see also Ranaee et al., 2019).

To summarize results of GSA, Principal Component Analysis (PCA) is applied to the desired responses of the MC reservoir simulations. This is conducive to Generalized Sensitivity Indices (GSI; Lamboni et al. (2011)) to quantify sensitivity of the reservoir simulation system (as a whole) to variations of the selected input parameters.

The manuscript is organized as follows. Section 2.1 introduces the experimental setup and conditions under which measurement-based information are collected. The direct pore-scale simulation technique (i.e., numerical approach) is illustrated in Section 2.2, generation of the heterogeneous fields for the reservoir model being presented in Section 2.3. Section 3 includes results of ML-based estimates of the controlling parameters and characterization of the related estimation confidence intervals. The implementation of such information for MC reservoir simulations is also illustrated. Finally, some key results of our numerical study are discussed in terms of the $(i)$ sensitivity and ( $i i)$ uncertainty of the reservoir simulation responses with respect to the uncertainty of the selected controlling parameters.

\section{Material and Methods}

Here, we present Steady-State (SS) two-phase coreflooding experiments (Moghadasi et al., 2015) as well as the methodology for the evaluation of two-phase relative permeabilities based on the use of porescale simulations (Guédon et al., 2019). We also describe the technique for the synthetic generation of heterogeneous fields (see Section 2.3) employed in our reservoir simulations.

\section{Experimental Setup and Conditions}

We perform our experiments on a Portland limestone core sample. A confining pressure of 90 bar is applied to stabilize the packing and prevent flow near the edges (see Moghadasi et al., 2019 for additional details). Table 1 lists some key attributes of the core samples.

Table 1-Attributes of the Portland core-sample

\begin{tabular}{|c|c|c|c|}
\hline $\begin{array}{c}\text { Length } \\
{[\mathrm{cm}]}\end{array}$ & $\begin{array}{c}\text { Diameter } \\
{[\mathrm{cm}]}\end{array}$ & $\begin{array}{c}\text { Absolute water permeability } \\
{[\mathrm{mD}]}\end{array}$ & $\begin{array}{c}\text { Porosity } \\
{[\%]}\end{array}$ \\
\hline 12.5 & 5.08 & 372 & 21 \\
\hline
\end{tabular}

A sodium Iodine (NaI) aqueous solution, crude oil from a Nigerian oil field and nitrogen (N2) are used during coreflooding as the water, oil, and gas phases, respectively.

The experimental setup consists of a hassler type core holder containing the sample placed in a saturation monitoring device. The latter relies on X-Ray attenuation to measure in-situ fluid saturation. The X-Ray apparatus includes a detector, a generator, and a motion-control system. A data acquisition system records temperature, pressure, flow rates, and X-Ray attenuation profiles. The system is designed as a semi-closed loop system, to allow for chemical equilibration between the fluids and the rock matrix, by recycling of the 
liquid phases. A detailed description of the experimental setup is detailed in our previous works together with the experimental procedure for data collection (Moghadasi et al., 2015).

Two-phase SS relative permeability experimental studies are performed by simultaneous injections of two fluids in the core. For two-phase water-oil experiments, data are collected by increasing the fractional flow of water while decreasing oil fractional flow to follow an imbibition path. A set of oil-gas drainage experiments is initiated through simultaneous flow of gas and oil at connate water saturation. During each experiment, the flow rate of the displacing phase (either water or gas) is increased while the flow rate of oil is decreased to follow saturation paths representing a reservoir behavior typically associated with twophase water-oil imbibition and gas-oil drainage (Moghadasi et al., 2017).

In the context of the SS technique, pressure gradient and saturations are measured at steady state conditions. Fluid properties, flow rates and pressure gradient are then employed to estimate effective permeabilities through the classical Darcy's equation.

Saturations of two-phase experiments are calculated by linear interpolation between the X-Ray attenuation coefficients associated with full sample saturation by oil, water, and gas. A detailed description of the methodology employed is presented in Moghadasi al. (2016).

Fig. 2 depicts two-phase relative permeability curves used for further applications at the field scale. Solid curves in Fig. 2 represent direct coreflooding data of $(i)$ imbibitions from two-phase water-oil environment and (ii) drainage under oil-gas conditions. The acquired laboratory data do not include a complete set of two-phase drainage/imbibition data, as one would require for the characterization of three-phase relative permeability models (eventually including hysteresis) to be employed in reservoir simulations. Accordingly, we rescale available data based on the values of saturation ending points (Schlumberger Geo-Quest, 2010), to evaluate two-phase relative permeability curves under oil-water drainage and gas-oil imbibition conditions (dashed curves in Fig. 2).
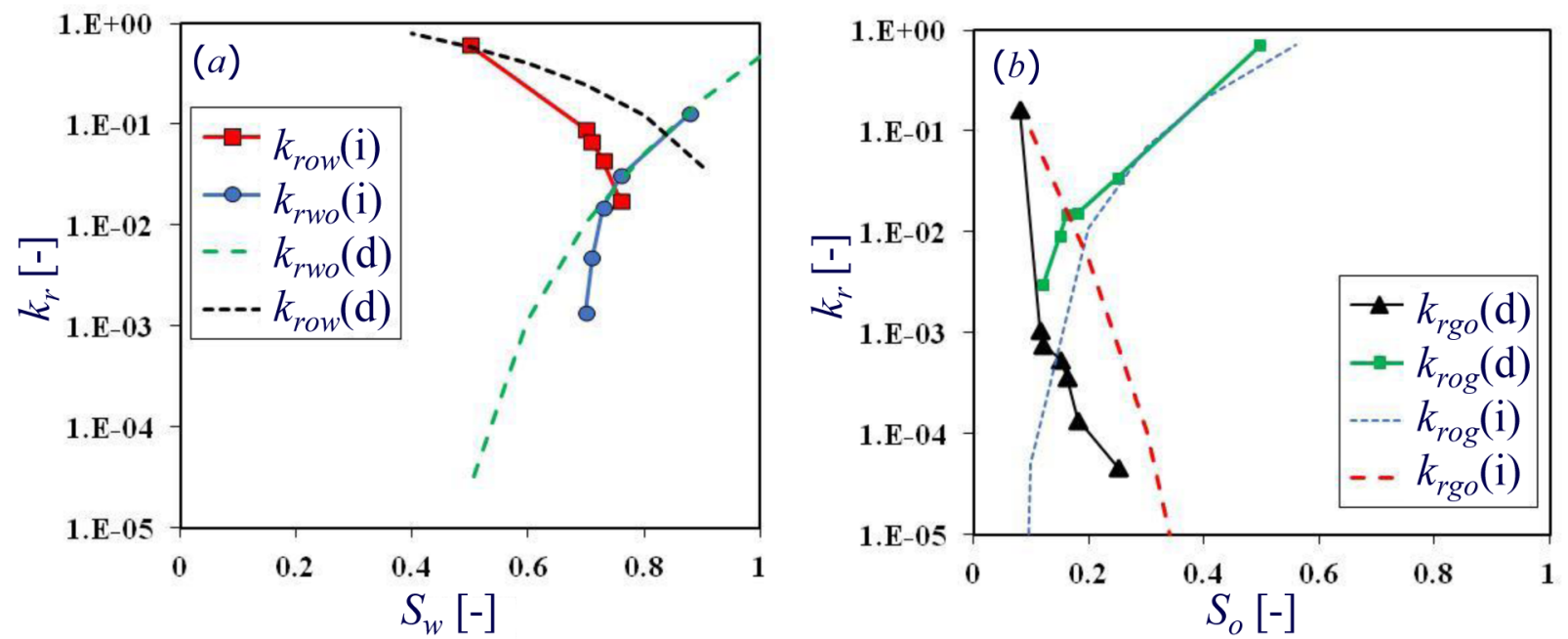

\begin{abstract}
Figure. 2-Laboratory data of two-phase relative permeabilities. Solid curves represent direct coreflooding data of imbibition in a two-phase (WO) environment and drainage under (OG) conditions. Dashed curves represent results obtained through rescaling of data (by considering saturation ending points) from two-phase (WO) environment and imbibition under (OG) conditions.
\end{abstract}

\title{
Pore-scale Simulations (Numerical approach)
}

A $1.2 \times 1.2 \times 1.8 \mathrm{~mm}^{3}$ tomographic image of the Portland core sample used during the experiments has been acquired for numerical analysis (see Guédon et al., 2019 for details). A collection of random realizations of the pore space is then generated to be employed in a numerical MC scheme. We do so upon relying on the technique introduced by Hyman and Winter (2014). The latter requires setting two parameters to obtain multiple realizations of the pore space, each characterized by the same porosity and correlation scale of 
the indicator function associated with the pore space of the target core sample. We extended this method by including an additional parameter to the generation procedure to enable controlling irregularities of pore shapes. We assess the ability of the realizations to mimic the Portland rock geometrical properties upon estimating the $(a)$ autocorrelation and $(b)$ lineal path functions associated with the pore space. The parameters of the generation are tuned until these functions obtained from the collection of realizations closely correspond to their counterparts obtained from the tomographic image. These are denoted as simulated and reference, respectively, in Fig. 3, where we depict a comparison between reference data and simulation results.

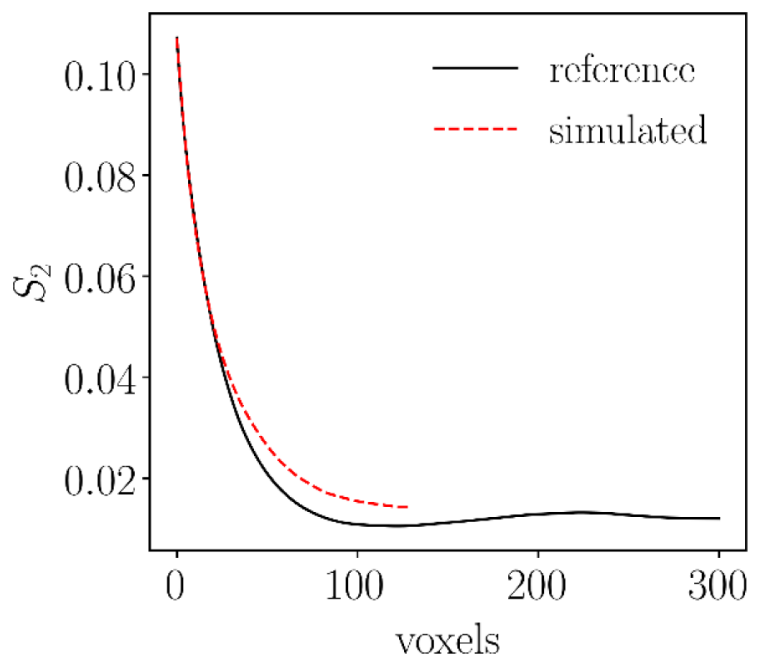

a) autocorrelation

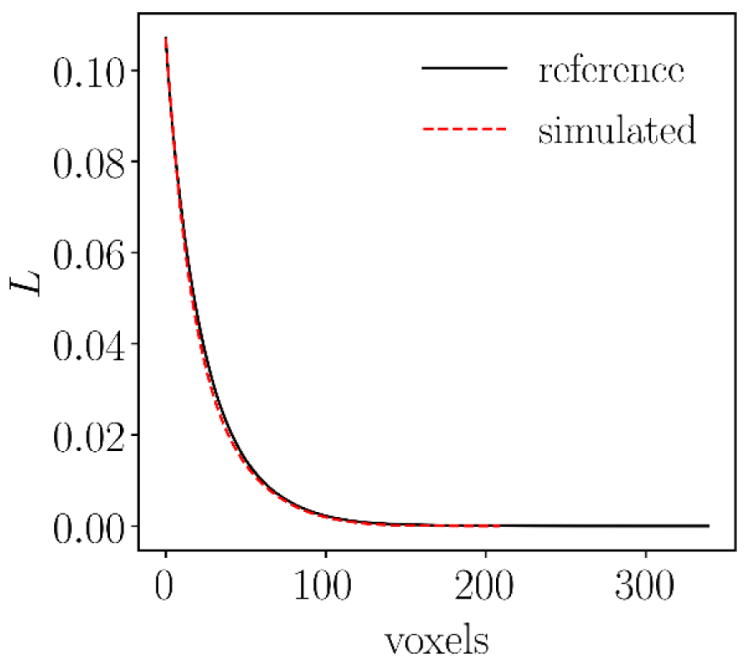

b) lineal-path

Figure 3-Comparison of (a) autocorrelation and (b) lineal-path functions obtained from reference (microCT scan of Portland rock) and simulated images (after tuning of the generation parameters).

Ten (random) samples have been considered and water-oil as well as oil-gas drainage and imbibition simulations have been performed (see Fig. 4). Simulation results are based on the use of the interFoam built-in solver of the OpenFOAM® open-source CFD toolbox (OpenCFD Limited., 2016). The latter employs a finite volume discretization of the Navier-Stokes equations and a Volume-of-Fluid technique to track interfaces between fluid phases. The numerical settings employed follow those implemented in our previous studies (Guédon et al., 2019). A Corey-type (Corey and Rathjens, 1956) two-phase relative permeability model is calibrated (through a ML approach, as detailed in Ranaee et al., 2016; 2017) to modeling results. The ensuing information has been used to determine confidence interval of the two-phase relative permeability curves and variance associated with estimates of the model parameters used in the MC simulations of the reservoir model illustrated in Section 3. 

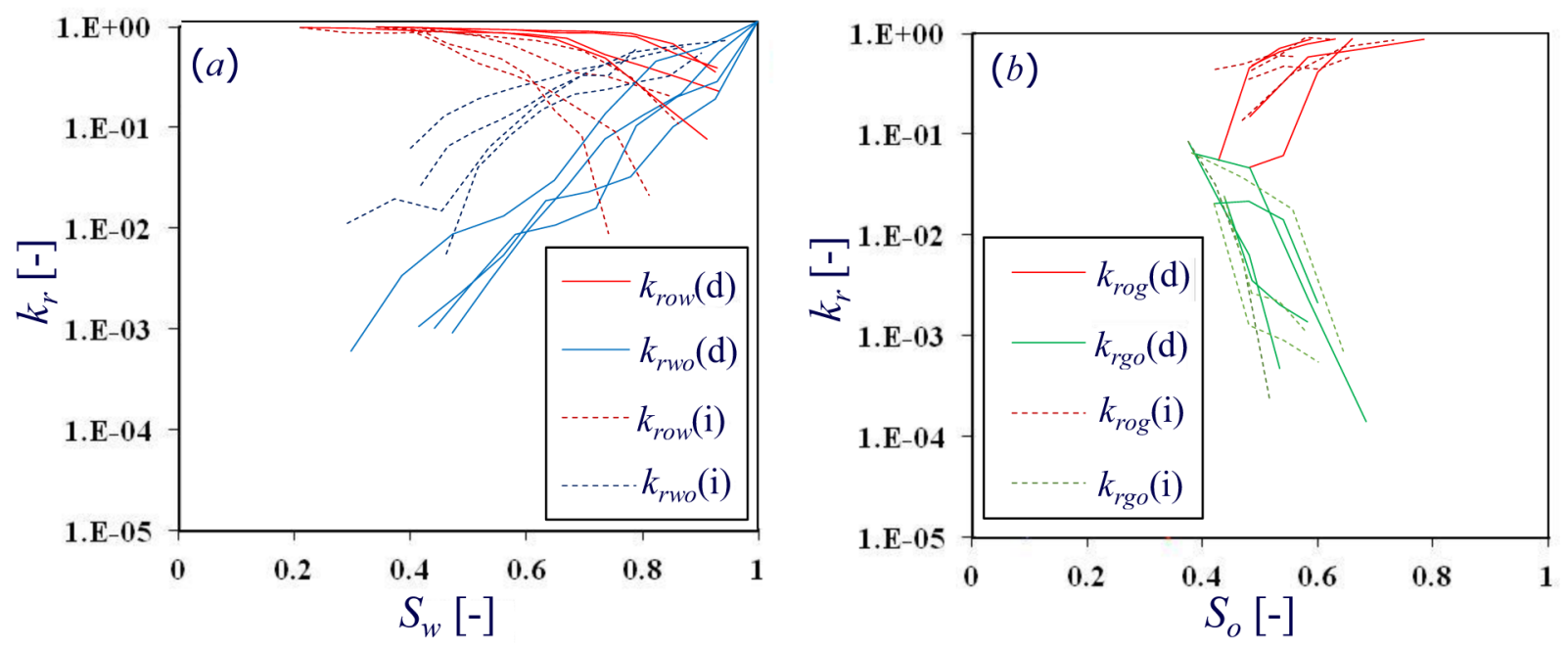

Figure. 4-Two-phase relative permeability curves obtained through the numerical approach for four selected (random) samples during $(a)$ WO drainage and imbibition and $(b)$ OG drainage and imbibition.

\section{Generation of Heterogeneous Fields of Reservoir Attributes}

Homogeneous fields are obtained upon assigning a spatially constant value of system attributes across the reservoir. Spatially heterogeneous distributions of attributes of the studied reservoir setting are generated according to the following steps:

a) generation of a random field, $u(x, y)$, associated with a uniform distribution (see Fig. 5a);

b) definition of a Gaussian kernel, $g(x, y)$, with intrinsic length scale $\lambda$ ( $\lambda=1$ in Fig. 5b);

c) convolution of $u(x, y)$ with $g(x, y)$ to obtain a correlated field $C_{\lambda}(x, y)$ (see Fig. 5c).

Fields where high values of permeability are well connected (corresponding to the heterogeneous and well-connected setting) are obtained upon implementation of the approach suggested by Zinn and Harvey (2003) to the fields generated as described above (see Fig. 5d). 
(a)

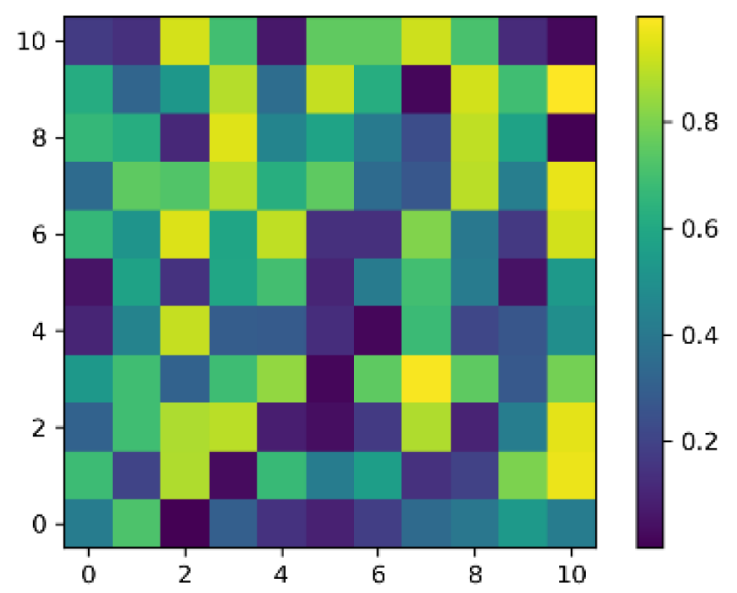

(c)

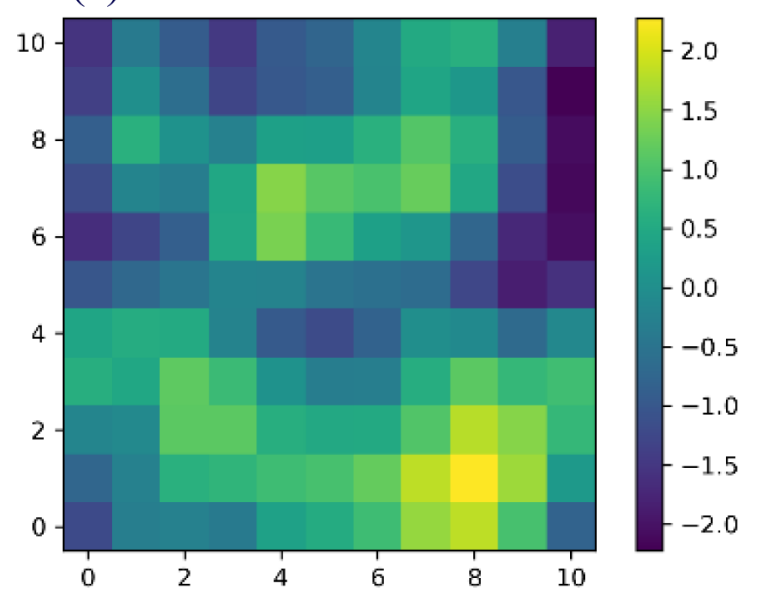

(b)

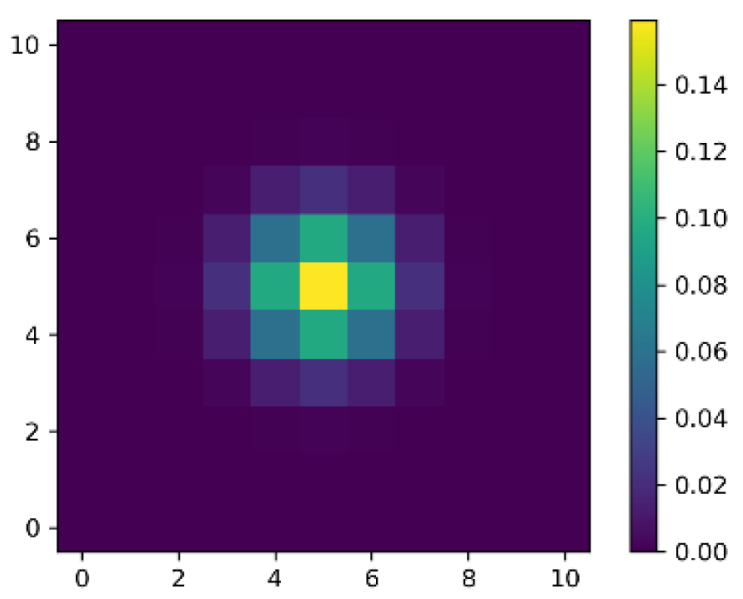

(d)

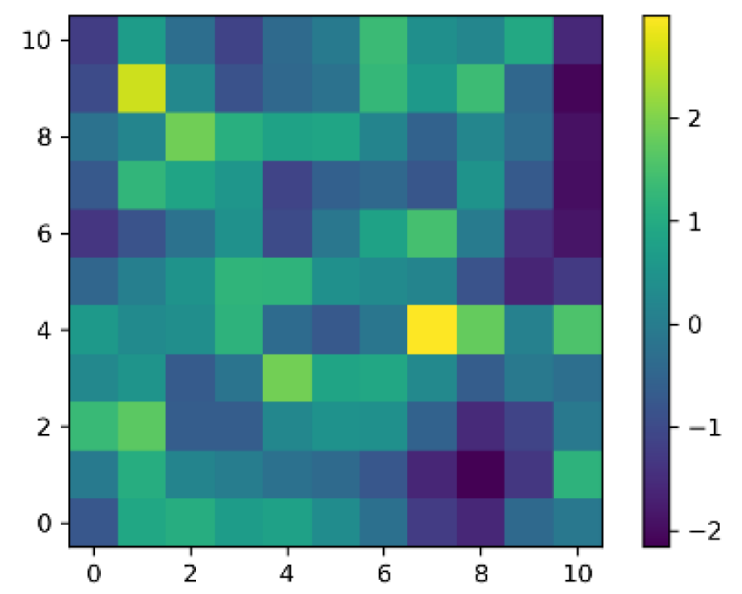

Figure. 5-Illustration of the heterogeneous fields generation: (a) a random field is generated, upon sampling from a uniform distribution; (b) a Gaussian kernel is defined, (c) the random field is convolved with the Gaussian kernel, and (d) the Zinn and Harvey (2003) approach is (optionally) applied to obtain a well-connected field.

\section{Results and Discussion}

\section{Setup of the Reservoir Model for MC Simulations}

We consider a two-dimensional reservoir model adapted from the original Odeh (1981) setup. The analysis is performed through the open source Matlab Reservoir Simulation Toolbox (MRST; see Lie (2019) for additional information). The computational domain is depicted in Fig. 6. 


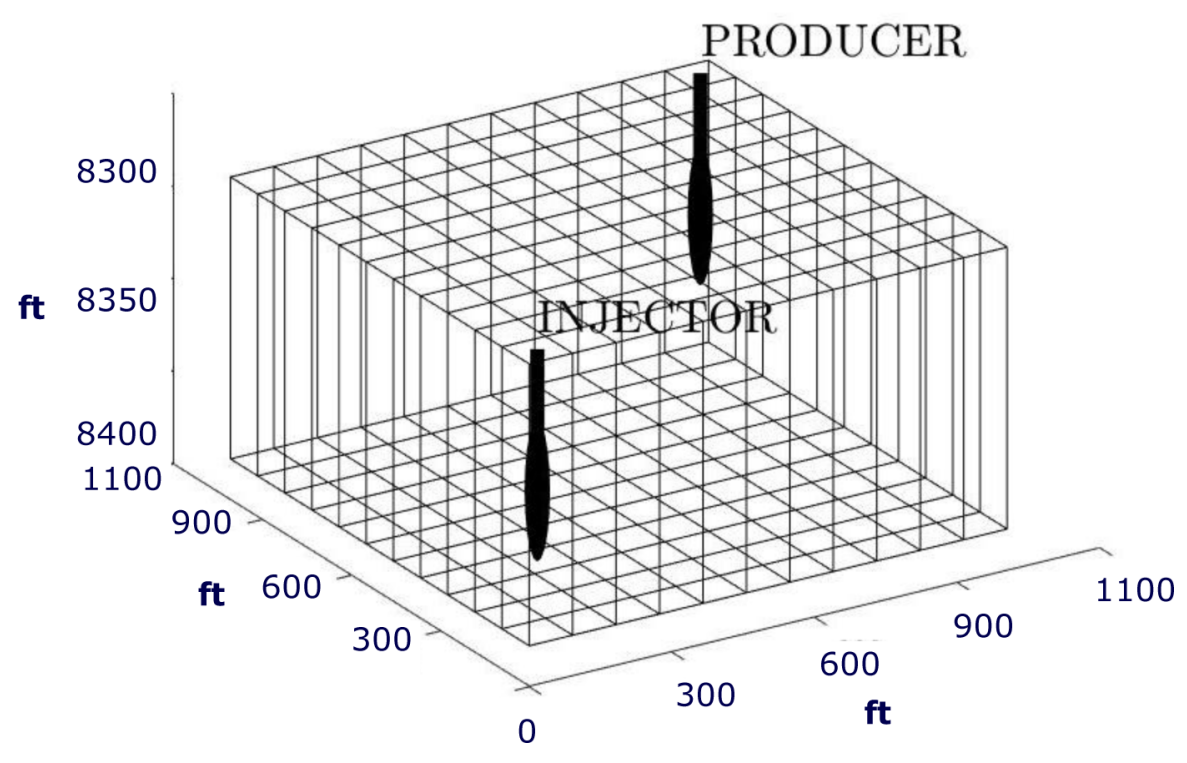

Figure. 6-Sketch of the reservoir simulation setup.

The reservoir has a size of $100 \times 100 \mathrm{ft}$ in the planar direction, with a thickness of $100 \mathrm{ft}$. The top of the reservoir is set at $8350 \mathrm{ft}$ below the surface level. The domain is discretized into 121 cells of uniform size (along the horizontal direction, see Fig. 6). Permeability and porosity distributions differ according to the setting analyzed, i.e., (a) homogeneous, (b) heterogeneous, or (c) heterogeneous and well-connected test cases (see Section 2.3 for details). Initial saturation conditions are set to connate water saturations (in the absence of gas), $S_{w c}$, which is evaluated according to the measurement-based information of Fig. 2 a.

The initial pressure is set to $4800 \mathrm{psia}$, the bubble point corresponding to $4014.7 \mathrm{psi}$. Densities of water, oil and gas at standard conditions are set to 64,50 and $1.2 \mathrm{lb} / \mathrm{ft} 3$, respectively, consistent with the coreflooding experiments. Viscosities of the fluids are set to $\mu_{w}=0.69 \mathrm{cP}, \mu_{o}=1.98 \mathrm{cP}$, and $\mu_{g}=0.0183 \mathrm{cP}$. Additional reservoir properties, as well as PVT characteristics of the fluids (live oil and dry gas) are set according to the original Odeh (1981) model. Injector/producer wells are located at the two opposite sides of the domain (see Fig. 6).

The injector well control is scheduled for alternating water and gas injection according to a yearly schedule up to a total of 25 years of production (i.e., 5 years of primary waterflooding followed by 20 years of WAG injection). Injection rate is set to $285 \mathrm{stb} /$ day and $198 \mathrm{Mscf} /$ day for water and gas, respectively. The producer well is set to $200 \mathrm{stb}$ /day liquid production while minimum bottom-hole pressure (BHP) is set to 1000 psi.

\section{Characterization of the Uncertain Model Parameters}

We consider the set of 15 parameters (collected in vector $\theta$ ) that we list in Table 2 according to the following categories: (i) hydraulic attributes (i.e., porosity, $\phi$, and absolute permeability, $k$ ) (ii) $\lambda$, representing the intrinsic length scale associated with the correlation of porosity and permeability, and (iii) twelve parameters associated with the Corey-type model (Corey and Rathjens, 1956) employed to represent two-phase WO and OG relative permeabilities.

The classical approach is typically based on information collected from direct coreflooding data from which one obtains parameter estimates (denoted as $\bar{\theta}$ in Table 2). While data are collected under a unique (one for WO and one for OG) saturation path (see Fig. 2), we consider here estimates to be associated with a standard deviation of $\pm 25 \% \bar{\theta}$ and values employed in the reservoir simulations to be randomly sampled from a uniform within the support $\bar{\theta} \pm 25 \% \bar{\theta}$ for the purpose of MC simulations. With reference to the numerical approach, values of $\theta$ are obtained from simulation results performed across ten realizations of the pore 
space (see Section 2.2). Symbols $\hat{\theta}$, and $\hat{\sigma}_{\theta}$ listed in Table 2 correspond to the mean and standard deviation, respectively, resulting from the analysis of such realizations. Note that, as opposed classical approach, the width of the interval associated with $\hat{\theta} \pm \hat{\sigma}_{\theta}$ is not uniform across the parameters $\left(\hat{\sigma}_{\theta}\right.$ varies between $1 \%$ and $163 \%$ of the value of $\hat{\theta}$ depending on the parameter considered).

Table 2-Table. 2 Characteristics of the model parameters evaluated through a single coreflooding experiment, $\bar{\theta}$, (classical approach) and associated standard deviation considered in the study. Mean value, $\hat{\theta}$, and standard deviation, $\hat{\sigma}_{\theta}$, resulting from 10 pore space simulations (numerical approach).

\begin{tabular}{|c|c|c|c|c|c|}
\hline \multirow{2}{*}{\multicolumn{2}{|c|}{ Parameters }} & \multicolumn{2}{|c|}{ Classical Approach } & \multicolumn{2}{|c|}{ Numerical Approach } \\
\hline & & \multirow{2}{*}{$\frac{\bar{\theta}}{0.123}$} & \multirow{2}{*}{$\frac{\bar{\sigma}_{\theta}}{ \pm 25 \% \bar{\theta}}$} & \multirow{2}{*}{$\frac{\hat{\theta}}{0.134}$} & \multirow{2}{*}{$\frac{\hat{\sigma}_{\theta}}{ \pm 15 \% \hat{\theta}}$} \\
\hline & $\phi$ & & & & \\
\hline & $k$ & $3.3 \times 10^{-13}$ & $\pm 25 \% \bar{\theta}$ & $3.7 \times 10^{-13}$ & $\pm 57 \% \hat{\theta}$ \\
\hline Intrinsic length scale & $\lambda$ & 1.5 & $\pm 25 \% \bar{\theta}$ & 1.5 & $\pm 25 \% \hat{\theta}$ \\
\hline \multirow{12}{*}{ Relative Permeability } & $S_{w c}$ & 0.4 & $\pm 25 \% \bar{\theta}$ & 0.33 & $\pm 45 \% \hat{\theta}$ \\
\hline & $S_{\text {row }}$ & 0.12 & $\pm 25 \% \bar{\theta}$ & 0.123 & $\pm 89 \% \hat{\theta}$ \\
\hline & $S_{\text {rog }}$ & 0.08 & $\pm 25 \% \bar{\theta}$ & 0.09 & $\pm 55 \% \hat{\theta}$ \\
\hline & $S_{g t}$ & 0.04 & $\pm 25 \% \bar{\theta}$ & 0.03 & $\pm 163 \% \hat{\theta}$ \\
\hline & $k_{r w o}^{\max }$ & 0.14 & $\pm 25 \% \bar{\theta}$ & 0.63 & $\pm 17 \% \hat{\theta}$ \\
\hline & $k_{\text {row }}^{\max }$ & 0.8 & $\pm 25 \% \bar{\theta}$ & 0.9 & $\pm 1 \% \hat{\theta}$ \\
\hline & $k_{\text {rog }}^{\max }$ & 0.71 & $\pm 25 \% \bar{\theta}$ & 0.78 & $\pm 30 \% \hat{\theta}$ \\
\hline & $k_{\text {rgo }}^{\max }$ & 0.165 & $\pm 25 \% \bar{\theta}$ & 0.163 & $\pm 46 \% \hat{\theta}$ \\
\hline & $n_{w o}$ & 5.5 & $\pm 25 \% \bar{\theta}$ & 1.4 & $\pm 50 \% \hat{\theta}$ \\
\hline & $n_{\text {ow }}$ & 1.7 & $\pm 25 \% \bar{\theta}$ & 1.1 & $\pm 68 \% \hat{\theta}$ \\
\hline & $n_{o g}$ & 3 & $\pm 25 \% \bar{\theta}$ & 2.2 & $\pm 36 \% \hat{\theta}$ \\
\hline & $n_{g o}$ & 12 & $\pm 25 \% \bar{\theta}$ & 1.3 & $\pm 62 \% \hat{\theta}$ \\
\hline
\end{tabular}

For reservoir simulation based on the numerical approach, we consider entries of $\boldsymbol{\theta}$ as independent and identically distributed (iid) random variables, each described by a Gaussian distribution, with mean value $\hat{\theta}$, and variance $\hat{\sigma}_{\theta}^{2}$ (see Table 2). We perform a set of $\mathrm{N}=5000 \mathrm{MC}$ simulations by sampling from the space of uncertain parameters $\theta$ and relying on the standard fully implicit finite volume formulation of black-oil reservoir simulation available in MRST. Model parameters for the heterogeneous scenarios are allowed to vary from one realization to another according to scenarios with various degrees of spatial heterogeneity, as driven by $\lambda$. Fig. 7 illustrates exemplary sample realizations of two-phase relative permeabilities. Fig. 8 represents exemplary sample realizations of porosity fields for the (a) heterogeneous and (b) well-connected heterogeneous field cases generated for MC reservoir simulations. 

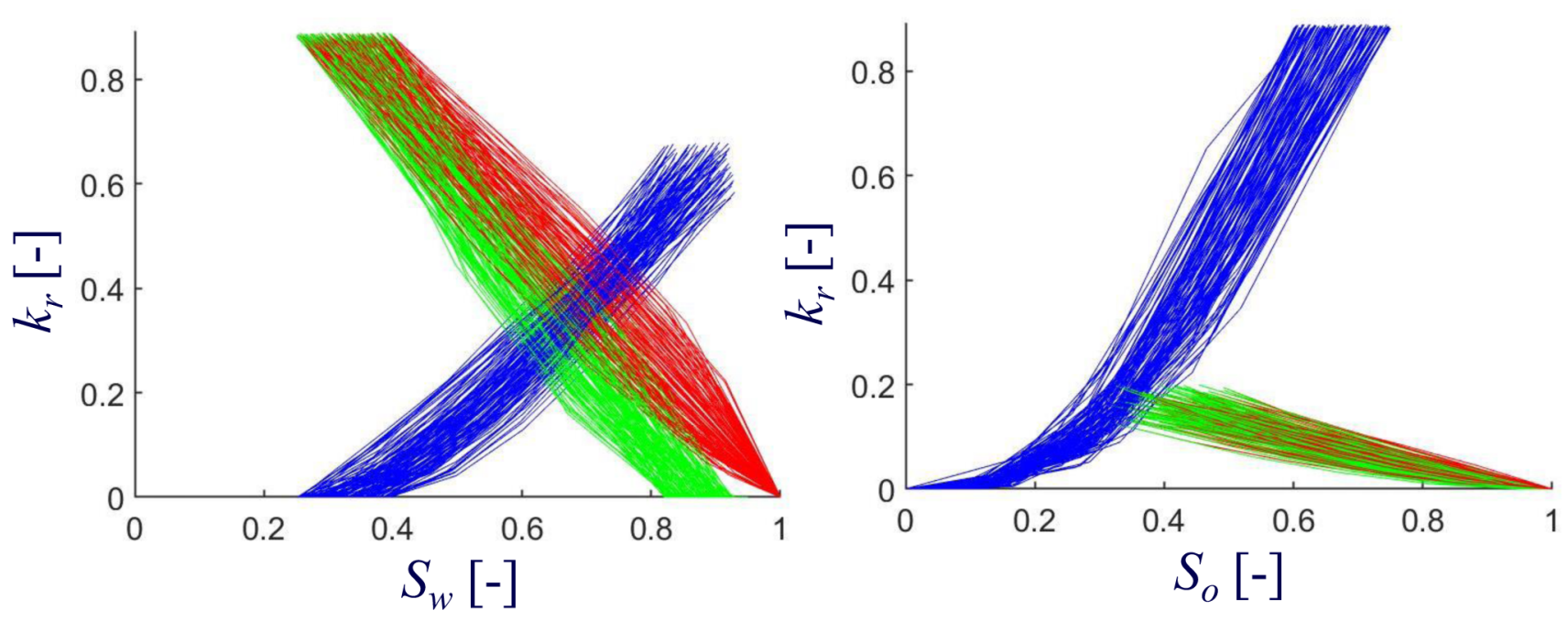

Figure. 7-Sample realizations of two-phase relative permeabilities used for MC reservoir simulations through the numerical approach.

(a)

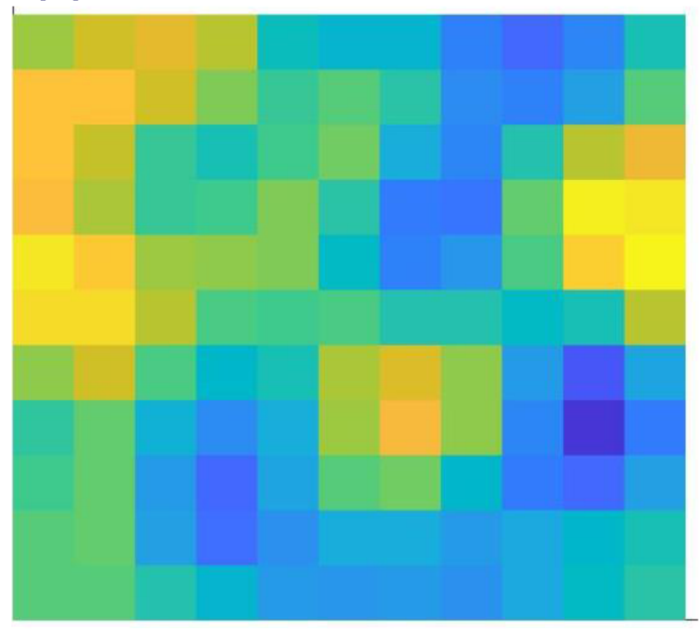

(b)

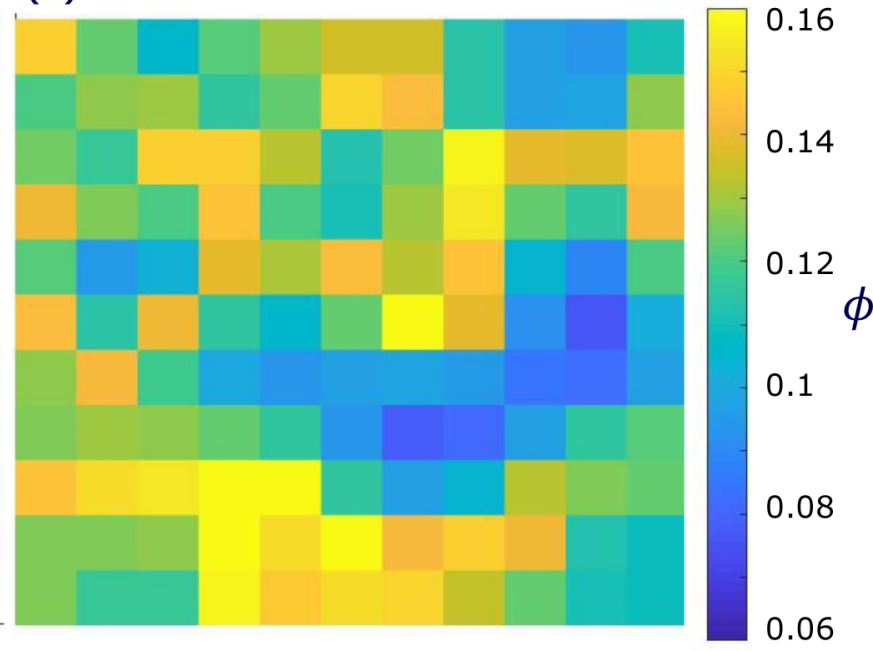

Figure. 8-Exemplary sample realizations of porosity generated for (a) heterogeneous and (b) well-connected heterogeneous field used for MC reservoir simulations.

Note that three-phase relative permeability, $k_{r \alpha}$ (with $\alpha=\mathrm{w}$, o, and g, referring to water, oil and gas, respectively), are modeled for the classical and numerical approaches by joint implementation of the Sigmoid-based oil relative permeability model (Ranaee et al., 2015) and of the Larsen and Skauge (1998) gas relative permeability hysteretic model, hysteresis effects on $k_{r w}$ being neglected, as we consider waterwet conditions.

\section{Sensitivity Analysis}

We rely on PCA-based GSA (in terms of Generalized Sensitivity Indices, GSI, detailed in the APPENDIX) to quantify the relative influence of the main system parameters (listed in Table 2) on key quantities (including field average pressure, FPR, water-cut, FWCT, gas-oil ratio, FGOR, and oil recovery, FOE) representing effectiveness of the WAG injection process. A numerical MC framework is employed to this end, the results illustrated in the following being associated with the numerical approach.

Fig. 9 represents the GSI evaluated for the variables listed Table 2. Here, $\mathbf{H}_{\mathrm{k}}$ represents reservoir simulation responses (in terms of the selected quantities), as a result of principal component analysis 
(Lamboni et al., 2011). Results are reported at the end of primary waterflooding (5 years of production) and at a five-year interval across the temporal window of WAG implementation (i.e., at 10, 15, 20, and 25 years of production). Here, porosity and absolute permeability fields are considered to be spatially homogeneous. These results suggest that the system is mainly sensitive to the curvature of the relative permeability curves during the early stages of production $\left(n_{w o}, n_{o g}\right.$, and $n_{g o}$ in Fig. $\left.9 \mathrm{a}\right)$. While production under WAG progresses, sensitivity of system responses to the values of saturation $\left(S_{w c}\right.$ and $S_{g t}$ in Fig. $\left.9 \mathrm{~b}-\mathrm{d}\right)$ and relative permeability $\left(k_{r w o}^{\max }\right.$ and $k_{r g o}^{\max }$ in Fig. $9 \mathrm{~b}$ to $\left.9 \mathrm{~d}\right)$ ending points tend to increase. Porosity almost dominates over the other variables within the last stages of production ( $\phi$ in Fig. 9e).

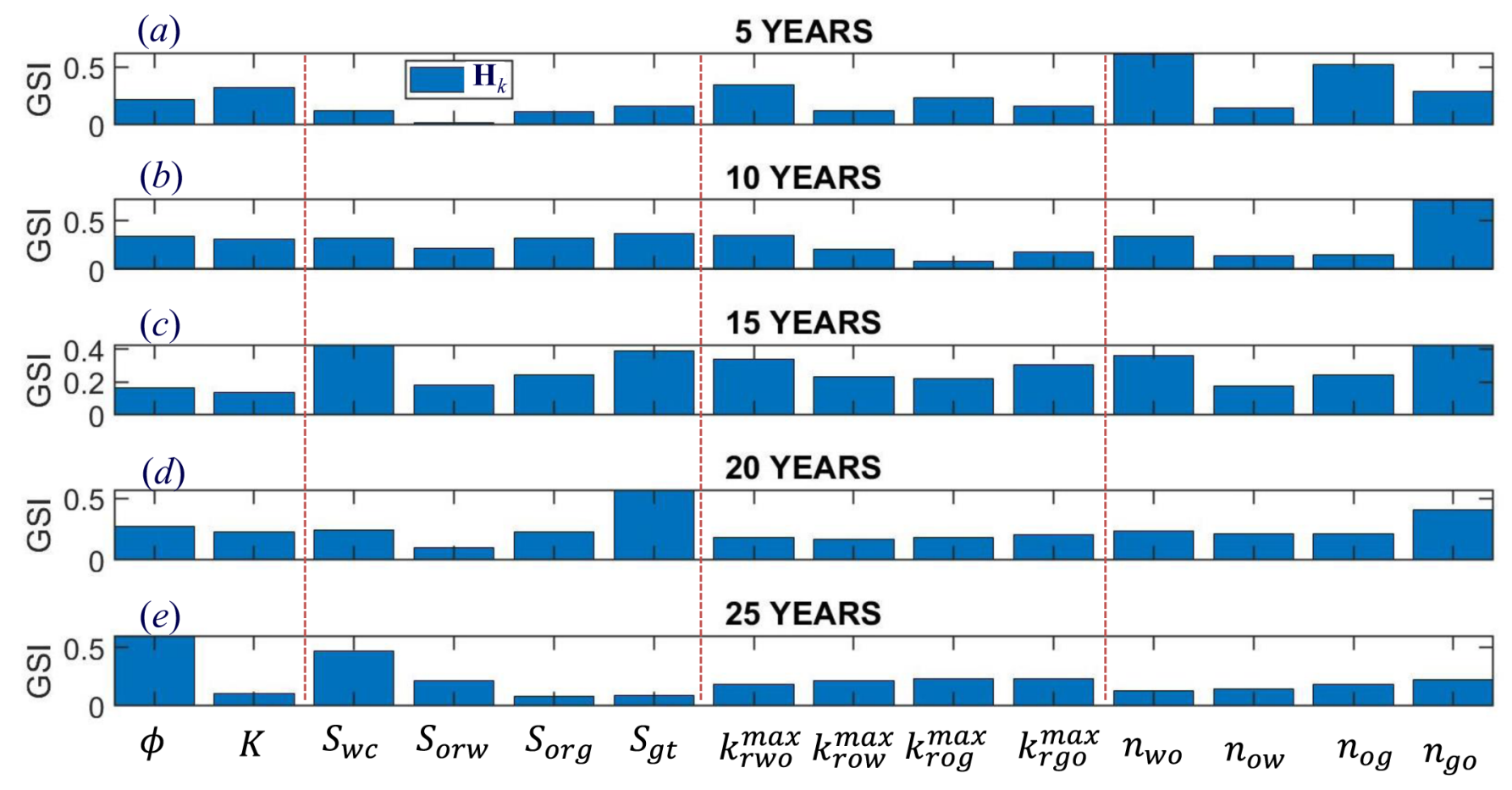

Figure. 9-Results of Global Sensitivity Analysis (in terms of GSI) of the reservoir simulation responses to the selected input variables. Results correspond to the numerical approach and are associated with $5,10,15,20$, and 25 years of production. Porosity and absolute permeability fields have been considered to be spatially homogeneous.

Fig. 10 illustrates corresponding results associated with the setting where porosity and absolute permeability fields are considered as spatially heterogeneous. At the first stages of production, porosity and parameters of the relative permeability model take an enhanced role to govern variations of system responses when the spatial distribution of the rock properties is treated to be heterogeneous. With the progress of production under WAG, sensitivity of the system responses to the values of absolute permeability increases. Simulation results show some mild sensitivity to the variation of the spatial heterogeneity of the reservoir properties (es expressed in terms of $\lambda$ ). 


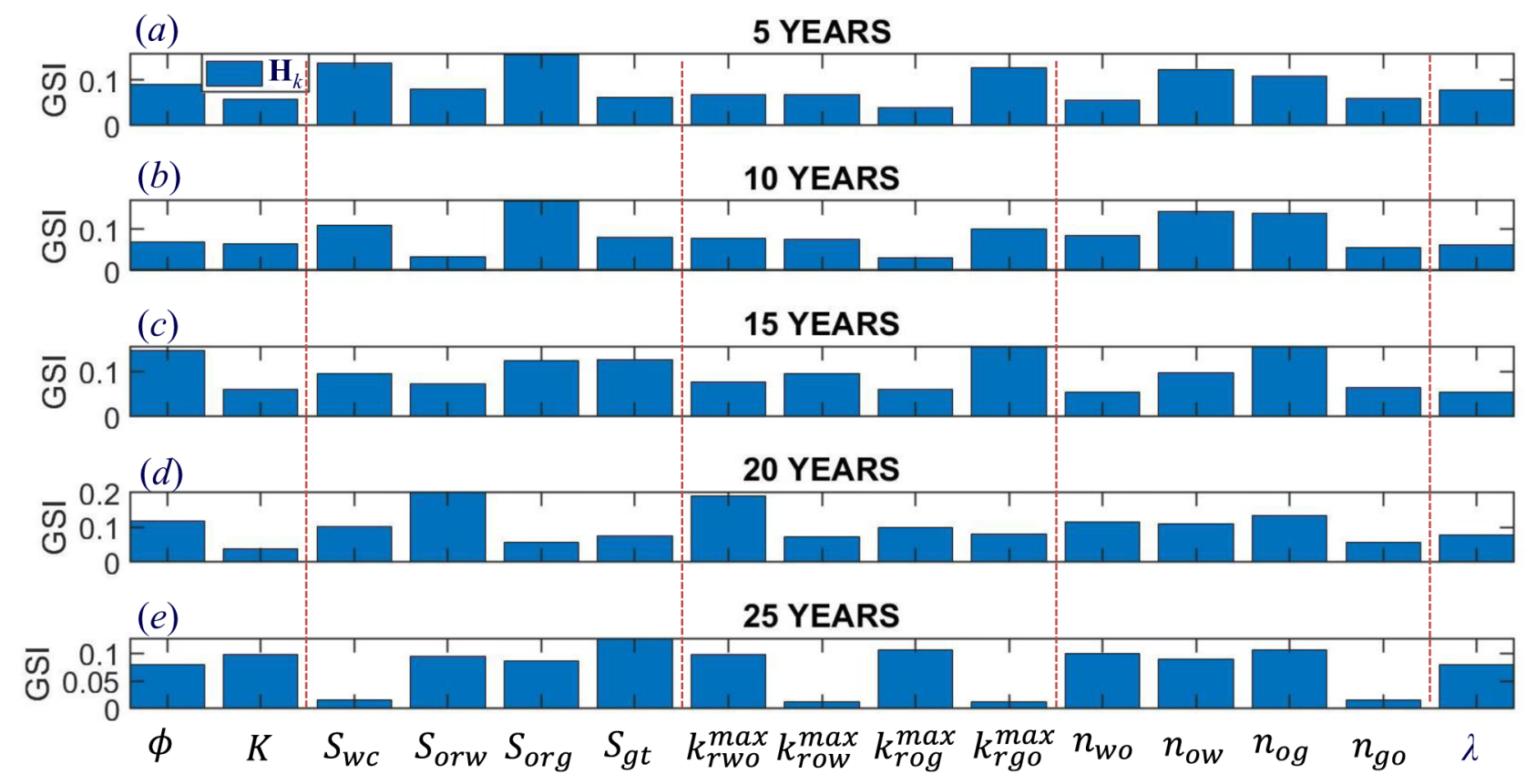

Figure. 10-Results of Global Sensitivity Analysis (in terms of GSI) of the reservoir simulation responses to the selected input variables. Results correspond to the numerical approach and are associated with 5, 10, 15, 20, and 25 years of production. Porosity and absolute permeability fields are considered to be spatially heterogeneous.

Fig. 11 illustrate results of the sensitivity analysis when porosity and absolute permeability are treated as heterogeneous and well-connected random fields. These results show that porosity dominates the remaining variables under primary waterflooding. Considering the evolution of the production under WAG, sensitivity of the system responses to the characteristics of the curvature of the relative permeability curves tends to increase. Such a behavior is in contrast to the results related to the homogeneous test case. Values of saturation and relative permeability ending points have only a secondary influence for the well-connected test case. An enhanced sensitivity to $\lambda$ is recognizable in Fig. 11, as compared against the results of Fig. 10 in the absence of connectivity. 


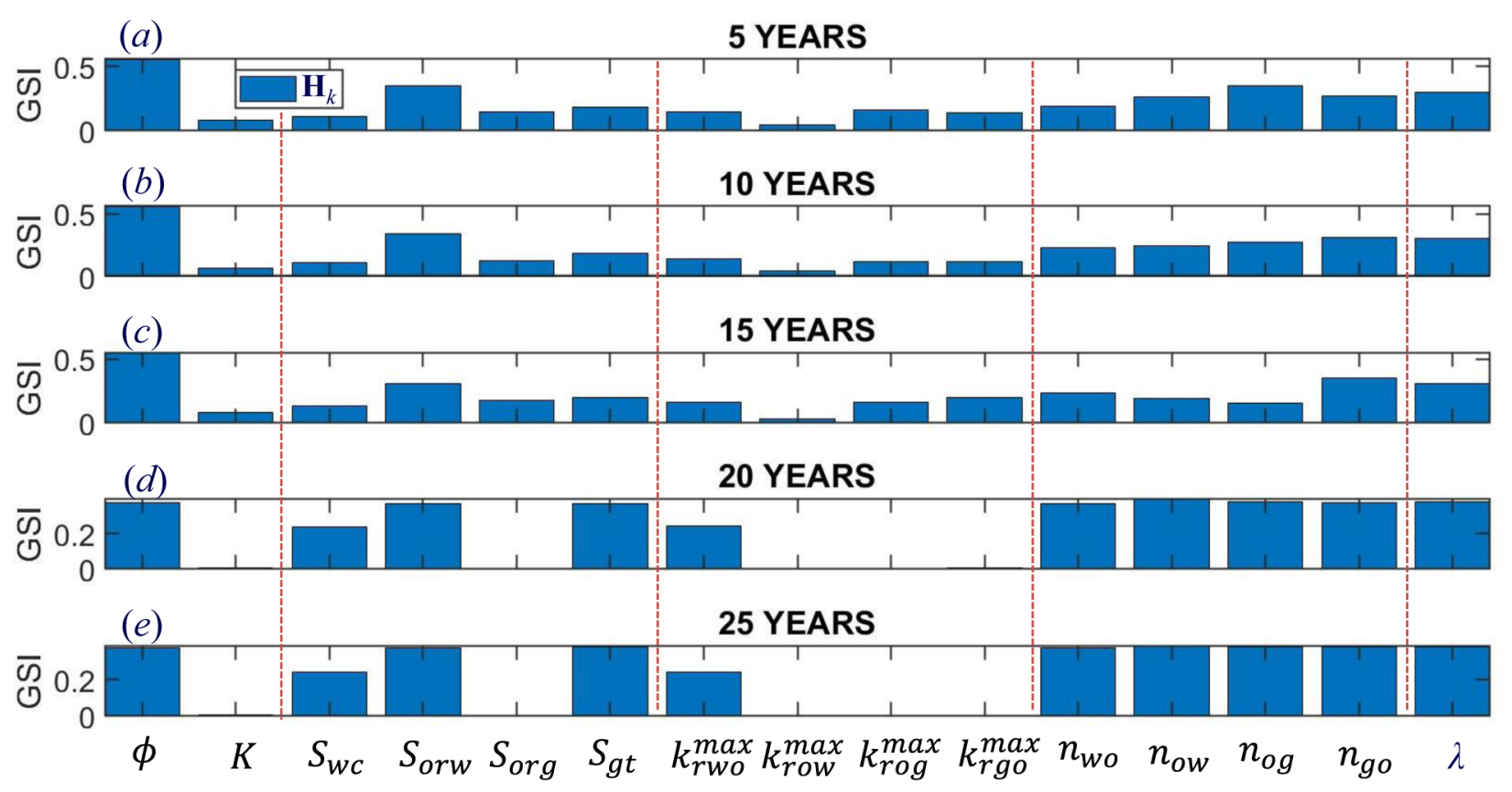

Figure. 11-Results of Global Sensitivity Analysis (in terms of GSI) of the reservoir simulation responses to the selected input variables. Results correspond to the numerical approach and are associated with 5, 10, 15, 20, and 25 years of production. Porosity and absolute permeability fields are considered to be spatially heterogeneous and well-connected.

\section{Uncertainty Assessment}

We investigate the way uncertainties associated with characterization of the parameters of the reservoir model propagate to the target responses of a reservoir simulation under WAG conditions. To summarize reservoir simulation results, Fig. 12 illustrates temporal evolutions of the NPV and the related uncertainty bounds (see Ranaee et al., 2018; 2019 for details of the analysis). Estimates of the mean, $\mu$, and the related uncertainty bound, $\pm \sigma$, are obtained from key statistics of the system responses from MC reservoir simulations (results are obtained through numerical (i.e., $\mu_{\mathrm{N}} \pm \sigma_{\mathrm{N}}$ ) and classical (i.e., $\mu_{\mathrm{C}}^{ \pm \sigma_{\mathrm{C}}}$ ) approaches).

In general, results show lower NPV values for the homogenous test case than for the heterogeneous scenarios. Uncertainty bounds with similar width (around the mean values) are found for all of the cases analyzed. Results obtained through the numerical approach clearly underestimates those evaluated through a classical approach. Note that $\mu_{\mathrm{N}}$ tends to stabilize approximately at the upper bound of uncertainty associated with the classical approach (i.e., $\mu_{\mathrm{C}}+\sigma_{\mathrm{C}}$ ) as time increases, and is always comprised within the range of $\mu_{\mathrm{C}} \pm \sigma_{\mathrm{C}}$. According to the results depicted in Fig. 12, NPV values evaluated through numerical and classical approaches show more similarities for the homogenous test case, as opposed to what can be observed for the heterogeneous and well-connected heterogeneous test cases. 


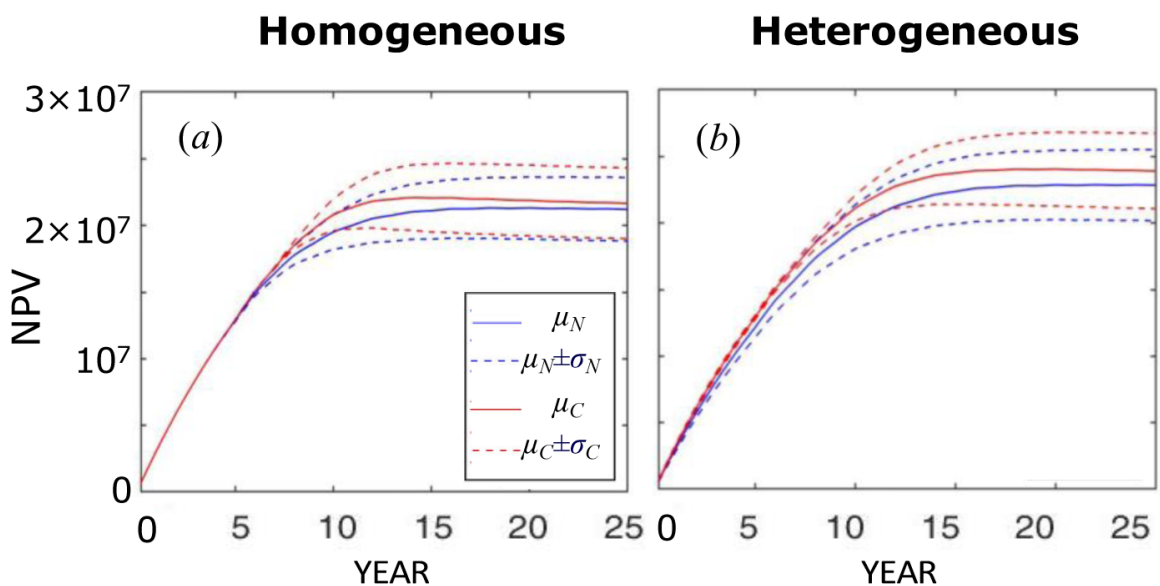

Figure. 12-Time evolution of net-present-value (NPV). Dashed curves represent uncertainty bound of width $\pm \sigma$ around the corresponding mean, $\mu$ (i.e., solid curves); $\mu_{\mathrm{C}}$ and $\mu_{\mathrm{N}}$ are evaluated through classical and numerical approaches, respectively. Results are evaluated through MC simulations and are depicted for (a) for homogeneous, (b) heterogeneous, and (c) well-connected heterogeneous fields.

\section{Conclusions}

A key outcome of the analysis is that sensitivity of the reservoir simulation results to the uncertainty in relative permeability/hysteresis model parameters depends on the degree of heterogeneity of the reservoir. Considering a homogeneous reservoir model, we note that the selected system responses (including FPR, FWCT, FGOR, and FOE) are mainly influenced by the characteristics of the relative permeability model in the early stage of production, the other parameters having only a secondary influence. Otherwise, system responses become mainly sensitive to porosity, $\phi$ and saturation ending points under WAG implementation. In the case of a heterogeneous reservoir, the situation changes and responses of the reservoir simulation are similarly influenced by porosity, relative permeability model parameters, and saturation ending points during the whole duration of production. In the case of well-connected heterogeneous fields, sensitivity to porosity is still high, the curvature of the relative permeability curves displaying an enhanced influence under WAG, as compared against the other scenarios. In this case, simulation results also show some sensitivity to the degree of the spatial heterogeneity of the field (as expressed by $\lambda$ ).

Our results suggest that approach based on pore-scale numerical simulation can be a potentially viable alternative to the classical approach of characterizing reservoir simulation model via coreflooding experiments. Hence, further investigations would be of interest to assess key parameters which are driving mean value (as well as other statistical moments) of reservoir simulation responses. Overall, we observe that the impact on reservoir outputs of uncertainties in the characterization of petrophysical properties (porosity/ permeability) can be stronger than or comparable to the effects of uncertainties associated with relative permeability model parameters. Such an impact is then driven by the degree of heterogeneity of the reservoir.

\section{Acknowledgements}

The authors are grateful to Eni SpA for granting permission to expose the results of these investigations.

\section{References}

Afzali, S., Rezaei, N., Zendehboudi S. [2018] A Comprehensive Review on Enhanced oil Recovery by Water Alternating Gas (WAG) Injection, Fuel, 227, 218-246, 10.1016/j.fue1.2018.04.015

Bianchi Janetti, E., Guadagnini, L., Riva, M., Guadagnini, A. [2019] Global Sensitivity Analyses of Multiple Conceptual Models with Uncertain Parameters Driving Groundwater Flow in a Regional-scale Sedimentary Aquifer. I HydroL, 574: 544-556. 10.1016/j.jhydro1.2019.04.035

Corey, A.T., Rathjens, C.H., Henderson, J.H., Wyllie, M.R.J. [1956] Three-Phase Relative Permeability. Journal of Petroleum Technology, 8 (11): 63-65. 10.2118/737-G 
Christensen, J.R., Stenby, E.H., Skauge, A. [2001] Review of WAG Field Experience. SPE-71203-PA, SPE Reservoir Eval. Eng., 4 (2) 19-23, 10.2118/71203-PA

Dell'Oca, A., Riva, M., Guadagnini, A. [2017] Moment-based Metrics for Global Sensitivity Analysis of Hydrological Systems. Hydrol. Earth SysL Sci., 21: 6219-6234. 10.5194/hess-21-6219-2017

Guédon, G.R., Inzoli, F., Riva, M., Guadagnini, A. [2019] Pore-scale Velocities in Three-dimensional Porous Materials with Trapped Immiscible Fluid. Physical Review E, 100: 043101. 10.1103/PhysRevE.100.043101

Hyman, J.D., Winter, C.L. [2014] Stochastic Generation of Explicit Pore Structures by Thresholding GaussianRandomFields,IComputationalPhysics, 277: 16-31. 10.1016/j.jcp.2014.07.046

Lamboni, M., Monod, H., Makowski, D. [2011] Multivariate Sensitivity Analysis to Measure Global Contribution of Input Factors in Dynamic Models. Reliability Engineering and System Safety, 96, 450-459. 10.1016/j.ress.2010.12.002

Larsen, J.A., Skauge, A. [1998]. Methodology for Numerical Simulation with Cycle-dependent Relative Permeabilities. 10.2118/38456-PA, SPE I, 3:(2) 163-173. 10.2118/38456-PA

Lie, K.A., [2019] An Introduction to Reservoir Simulation Using MATLAB/GNU Octave: User Guide to the MATLAB Reservoir Simulation Toolbox (MRST), Cambridge University Press, 10.1017/9781108591416

Moghadasi, L., Ranaee, E., Renna, D., Bartosek, M., Maddinelli, G., Masserano. F., Cominelli, A., Inzoli, F., Guadagnini, A. [2020] Combining Two- and Three-Phase Coreflooding Experiments for Reservoir Simulation under WAG Practices. IPTC-19914-Abstract, Presented at International Petroleum Technology Conference, 13-15 January, Dhahran, Kingdom of Saudi Arabia. 10.2523/IPTC-19914-Abstract

Moghadasi, L., Renna, D., Bartosek, M., Maddinelli, G., Scagliotti. S. [2019] Experimental Investigation of Three-Phase Relative Permeability under Simultaneous Water and Gas (SWAG) Injection. Presented at 20th European Symposium on Improved Oil Recovery, 8 April, Pau, France. 10.3997/2214-4609.201900078

Moghadasi, L., Inzoli, F., Guadagnini, A., Renna, D., Bartosek, M., Maddinelli, G. [2017] Use of X-Ray Saturation Measurements in Flow through Investigations for the Characterization of Two- and Three-Phase Relative Permeability of Carbonate Rock. OMC-2017-774, Presented at Offshore Mediterranean Conference and Exhibition, Ravenna, Italy.

Moghadasi, L., Guadagnini, A., Inzoli, F., Bartosek, M., Renna, D. [2016] Characterization of Two- and Three-Phase Relative Permeability of Water-Wet Porous Media through X-Ray Saturation Measurements. 1 Petrol. Sci. and Eng., 145: 453-463. 10.1016/j.petro1.2016.05.031

Moghadasi, L., Guadagnini, A., Inzoli, F., Bartosek, M. [2015] Interpretation of Two-Phase Relative Permeability Curves through Multiple Formulations and Model Quality criteria. I Petrol. Sci. and Eng., 135: 738-749. 10.1016/ j.petro1.2015.10.027

Odeh, A. [1981] Comparison of Solutions to a Three-Dimensional Black-oil Reservoir Simulation Problem. SPE-9723PA, I Petrol. Technol, 33 (1): 13-25. 10.2118/9723-PA

OpenCFD Limited. [2016] OpenFOAM The Open Source CFD Toolbox, user guide version v1612, https:// www.openfoam.com/(2016).

Ranaee, E., Inzoli, F., Riva. M., Guadagnini, A. [2019] Hysteresis Effects of Three-phase Relative Permeabilities on Black-oil Reservoir Simulation under WAG Injection Protocols. J. Petrol. Sci. and Eng., 176: 1161-1174, 10.1016/ j.petro1.2019.01.044

Ranaee, E., Inzoli, F., Riva, M., Cominelli, A., Guadagnini, A. [2018] Propagation to Reservoir Simulation of Uncertainty Associated with Three-phase Relative Permeability Models with Hysteresis, SPE-190825-MS, Presented at SPE Europec featured at 80th EAGE Conference and Exhibition, 11-14 June, Copenhagen, Denmark. 10.2118/190825-MS

Ranaee, E., Moghadasi, L., Inzoli, F., Riva, M., Guadagnini, A. [2017] Identifiability of Parameters of Three-phase oil Relative Permeability Models under Simultaneous Water and Gas (SWAG) Injection. Petrol. Sci. and Eng., 159: 942-951, 10.1016/j.petro1.2017.09.062

Ranaee, E., Riva, M., Porta, G.M., Guadagnini, A. [2016] Comparative Assessment of Three-phase oil Relative Permeability Models. In: Water Resour. Res., 52 (7) 5341-5356, 10.1002/2016WR018872

Ranaee, E., Porta, G.M., Riva, M., Blunt, M.J., Guadagnini, A. [2015] Prediction of Three-phase Oil Relative Permeability through A Sigmoid-Based Model. In: I Petrol. Sci. and Eng., 126, 190-200, 10.1016/j.petro1.2014.11.034

Saltelli, A., Ratto, M., Andres, T., Campolongo, F., Cariboni, J., Gatelli, D., Salsana, M., Tarantola, S. [2008] Global Sensitivity Analysis - The primer. Wiley.

Schlumberger Geo-Quest. [2010] ECLIPSE 100 Reference Manual.

Sobol, I. [1993] Sensitivity Analysis for Non-linear Mathematical Models. Mathematical Modeling and Computational Experiments. 1 (4), 407-414. 10.1016/S0378-4754(00)00270-6

Zinn, B., Harvey, C.F. [2003] When Good Statistical Models of Aquifer Heterogeneity go bad: A Comparison of Flow, Dispersion, and Mass Transfer in Connected and Multivariate Gaussian Hydraulic Conductivity Fields. Water Resour. Res., 39 (3): 1051, 10.1029/2001WR001146 


\section{APPENDIX. PCA-based Sensitivity Indices}

By combining the principal component decomposition of the simulation outputs $\boldsymbol{Y}$ and the analysis of variance (ANOVA) decomposition, Lamboni et al. (2011) define a set of sensitivity indices associated with the principal components of the whole multivariate outputs.

Following Lamboni et al. (2011), we first perform PCA through the eigenvalue decomposition of the covariance matrix of the system outputs $\boldsymbol{Y}$ and transform the model outputs into a set of new orthogonal variables, which are sorted according to strength of their contribution to the overall variance.

To this end, values of the outputs $\boldsymbol{Y}$ are normalized and we denote the (mean-centered) centered outputs as $\boldsymbol{Y}^{c}$, i.e.,

$$
\mathbf{Y}^{c}=\mathbf{Y}-\boldsymbol{\mu}_{\mathbf{Y}}
$$

where $\boldsymbol{\mu}_{\boldsymbol{Y}}$ is the vector of the mean outputs.

We then perform the eigenvalue decomposition of the covariance matrix, i.e.,

$$
\Sigma_{\mathbf{Y}}=\Gamma \Lambda \Gamma^{\mathbf{T}}
$$

where $T$ denotes transpose, $\Sigma_{\boldsymbol{Y}}$ is the covariance matrix of outputs $\boldsymbol{Y}, \boldsymbol{\Lambda}=\operatorname{diag}\left(\lambda_{1}, \ldots, \lambda_{m}\right)$ is the diagonal eigenvalue matrix (i.e. $\lambda_{1} \geq \lambda_{1} \geq \ldots \geq \lambda_{\mathrm{m}}$ are the eigenvalues), $\Gamma=\left(\gamma_{1}, \gamma_{2}, \ldots, \gamma_{\mathrm{m}}\right)$ is the eigenvector matrix, $\gamma_{\mathrm{i}}$ (with $i=1,2, \ldots, \mathrm{m}$ ) represent the normalized and mutually orthogonal eigenvectors associated with the eigenvalues. The centered outputs, $\boldsymbol{Y}^{c}$, are then transformed into independent variables $\boldsymbol{H}$ through

$$
\mathbf{H}=\mathbf{Y}^{C} \boldsymbol{\Gamma}
$$

where $\boldsymbol{H}=\left(\boldsymbol{H}_{1}, \boldsymbol{H}_{2}, \ldots, \boldsymbol{H}_{m}\right)$ contains the principal components, which are orthogonal to each other. $\boldsymbol{H}_{\boldsymbol{m}}$ (with $k=1,2, \ldots, \mathrm{m}$ ) is centered (around the mean values of $\mu_{Y}$ ) with variance $\lambda_{k}$, i.e., $V\left(\mathbf{H}_{m}\right)=E\left(\mathbf{H}_{k}^{2}\right)=\lambda_{k}$. We note that $\sum_{k=1}^{m} \lambda_{k}=$ trace $\left(\Sigma_{\mathbf{Y}}\right)$. Through Eq. (A.3), $\boldsymbol{Y}^{c}$ can also be expressed by $\boldsymbol{H}$ as

$$
\mathbf{Y}^{C}=\mathbf{H} \boldsymbol{\Gamma}^{\prime}
$$

where $\boldsymbol{\Gamma}$ represents the inverse of matrix $\boldsymbol{\Gamma}$. Thus, the original outputs $\boldsymbol{Y}$ can be expressed in terms of the mutually orthogonal principal components in $\boldsymbol{H}$ as

$$
\mathbf{Y}=\boldsymbol{\mu}_{\mathbf{Y}}+\mathbf{H} \boldsymbol{\Gamma}^{\prime}
$$

Only the first $K$ principal components containing the highest contribution to the variance of the original outputs are typically retained. Then, $\boldsymbol{Y}$ can be approximated as

$$
\mathbf{Y}=\boldsymbol{\mu}_{\mathbf{Y}}+\mathbf{H}_{K} \boldsymbol{\Gamma}_{K}^{\prime}
$$

Here $\boldsymbol{H}_{K}$ contains the first $K$ principal components of $\boldsymbol{H}, \boldsymbol{\Gamma}_{K}$ denoting the first $K$ eigenvectors in $\boldsymbol{\Gamma}$.

Lamboni et al. (2011) defined a set of sensitivity indices on the principal components $H_{K}$ (with $k=1,2$, $\ldots, m)$ across the whole multivariate outputs.

We consider here solely the first order sensitivity index of model input variable on the k-the principal component $\boldsymbol{H}_{K}$ of the multivariate outputs $\boldsymbol{Y}$, which is defined as

$$
S I_{i, k}=\frac{V_{i, k}}{V_{k}}
$$

where $V_{i, k}$ is the variance of $H_{k}$ due to variability of input variable $X_{i}$ and $V_{k}=\lambda_{k}$ is the variance of $\boldsymbol{H}_{k}$. The above sensitivity indices focus on the $K$-th principal component of model outputs rather than on the whole multivariate collection of outputs. To quantify the contribution of each input variable $X_{i}$ to the total variance of the multivariate outputs $\boldsymbol{Y}$, Lamboni et al. (2011) further define the generalized first order sensitivity index as 


$$
G S I_{i}=\Sigma_{k=1}^{K} \frac{\lambda_{k}}{\sum_{i=1}^{m} \lambda_{l}} S I_{i, k}
$$

where $\lambda_{k}$ is the $K$-th eigenvalue (the variance of the principal component).

The generalized first order sensitivity index $\boldsymbol{G S I}_{\boldsymbol{i}}$ embeds the overall contribution of the input variable $\boldsymbol{X}_{\boldsymbol{i}}$ on the total variance of the multivariate outputs $\boldsymbol{Y}$ (as a whole). A generalized sensitivity index is then interpreted as the weighted average of the sensitivity indices on the principal components, and the weight of each term is proportional to the eigenvalue of the corresponding principal components. 